\title{
Trust and Culture in Rural Research Design
}

\author{
Lacey Andrews ${ }^{1}$, Joy Butcher-Winfree ${ }^{2}$, Marianna Linz ${ }^{3}$, Todd Davies ${ }^{4}$, * \\ ${ }^{1}$ Department of Pediatrics, Vanderbilt University Medical Center, Nashville, Tennessee \\ ${ }^{2}$ Behavior Health Department, Tug River Health Association, Gary, West Virginia \\ ${ }^{3}$ Psychology Department, Marshall University, Huntington, West Virginia \\ ${ }^{4}$ Department of Family and Community Health, Joan C Edwards School of Medicine, Marshall University, Huntington, West Virginia
}

Email address:

daviest@marshall.edu (T. Davies)

${ }^{*}$ Corresponding author

\section{To cite this article:}

Lacey Andrews, Joy Butcher-Winfree, Marianna Linz, Todd Davies. Trust and Culture in Rural Research Design. American Journal of Health Research. Vol. 9, No. 3, 2021, pp. 64-71. doi: 10.11648/j.ajhr.20210903.11

Received: April 15, 2021; Accepted: May 5, 2021; Published: May 14, 2021

\begin{abstract}
Background: Community isolation, poor economic conditions, intimate group structures and the natural terrain creates unique challenges in both the treatment and study of opioid use disorder (OUD). The maternal and neonatal consequences for OUD can be profoundly altered by co-occurring psychiatric conditions in the mother, a phenomenon that may be more profound in rural communities. Because of these unique issues, the level of direct interaction in a patient-centric rural research design is critical to the effectiveness of the study. Objective: We set out to test the hypothesis that maternal stress and psychological state has a prolonged developmental impact on prenatally exposed children in rural areas by reducing needed stimulation in the caretaking environment. The study quickly changed as we discovered the geographic isolation and health disparate nature of the community had an important implication for research design and data collection. Methods: We used a typical patient-centered study design method for an observational study in rural West Virginia. Results: Original concise designs created complications for participants leading to recruitment difficulties and poor retention. Patients were resistant to study participation related to culture and severe community health disparities that were not identified until the patient population became comfortable with the research team. Conclusion: Despite the cultural and genetic background similarities between patients in less isolated areas and the test site, the geographic isolation and health disparate nature of the community had a profound effect on the research design. The findings in this study suggest a reevaluation of approaches to conducting research in rural isolated areas.
\end{abstract}

Keywords: NAS, MAT, OUD, Rural, Research Design

\section{Introduction}

Research providing guidance and best practices for identifying, recruiting, and retaining participants in research studies is limited. [1-8] Recently there has been an increase in "patient centric" trial designs that are inclusive of potential participants needs and expectations early in the design phases as described by Sharma [9] and Lim et al. [10] Although novel, patient centric study designs have increased efficacy of trials in some cases. $[9,10]$ However, the vast majority of "patient centric" trials have occurred in urban areas.

Research in urban areas flourishes due in part to larger populations aiding recruitment, public familiarity with researchers and research procedures, as well as readily available resources. Patient centric research designs in theory will only strengthen the success of research in urban areas as participants help increase study efficacy, recruitment and retention. However, research in rural resource poor areas is often more complicated. Overwhelming epidemics such as the opioid epidemic places a further strain on rural research projects. While patient centric research designs can help elevate some strain, trust may be the main component for a successful research trial in rural areas. Gaining participants trust may potentially blur common ethical strategies, take a considerable amount of time, and aid in budget conflicts as illustrated by the complications of the following research study focused on opioid use disorder (OUD).

Objective 
The opioid epidemic is a primary public health issue disproportionally affecting low resource rural communities. [11-15] OUD takes root in small communities and spreads rapidly due to the intimacy of the group structure. In addition to aiding the spread of OUD, intimate group structures can act as triggers for relapse during recovery periods. Community isolation, group structure, poor economic conditions, and natural terrain creates distinct challenges in both the treatment and study of OUD. These characteristics not only effect OUD but can also greatly impact study design and data collection further convoluting rural research projects.

OUD is often a part multiple complex behavioral health issues, of which the most common co-occurring disorders being depression and anxiety. Both common disorders are linked with several adverse birth and developmental outcomes. $[16,17]$ The consequences of opioid exposure and withdrawal in infants can be profoundly exacerbated by the presence of these co-occurring disorders in the mother. Known adverse outcomes include: preterm labor, low birth weight, delayed pediatric psychomotor skills, behavioral difficulties and neonatal abstinence syndrome (NAS) as a result of self-medication through opioid use. [18, 19] It has also been reported that maternal stress and psychological state may have a prolonged developmental impact by reducing needed stimulation in the caretaking environment. [20] The effect may be more profound with women in rural areas as they have a high reliance on community structures which can be disrupted due to social withdrawal during an increase in depressive symptoms. We set out to test this hypothesis through an observational research study of pregnant women participating in the medication assisted treatment (MAT) program in a rural, resource poor area in southern West Virginia. Despite a similar cultural and genetic background between patients in the less isolated areas of the State and the test site, we discovered the geographic isolation and health disparate nature of the community had an important implication for research design and data collection. Furthermore, we discovered unique complication of conducting rural research with a vulnerable population.

\section{Methods}

During the study design process, professionals from our institution and the rural test site collaborated extensively on the study design to ensure feasibility and appropriateness. These design meetings not only establish an invaluable working relationship between both institutions but also provided our researchers with a better understanding of the rural population. The insight provided from the test site led to a more patient focused research design helping researchers consider unique obstacles this population would face while participating in a research study. The most common obstacles were transportation issues, time constraints, and a general lack of understanding of research logistics and health needs that exceeded OUD. While participants did not provide the information for the original design, the information was provided from a source that knew the patient population exceedingly well and understood the obstacles the population faced daily to receive treatment for OUD. However, we did not anticipate our patient focused research design would cause more issues than it solved. Nor did we foresee obstacles such as fear, of which had the greatest impact on recruitment and retention. Both obstacles illustrate the importance of trust and a true patient centric study design

\section{Results}

Collaboration efforts shaped the original project design into what we considered realistic for the population. However, once initiated the original concise design (Figure 1) created complications for participants leading to recruitment difficulties and poor retention. Those issues were taken in stride and efforts were made to correct them after receiving vital participant input. Counter to our original assumptions a more complex visit schedule (Figure 2) was more effective than the original design. While a positive impact was made on our study and patient centric study designs are becoming well adapted and accepted, our recommended approach may raise many ethical considerations as well as time constraints and budget concerns.

\section{Discussion}

The suggested approach raises many ethical considerations including dual relationships, coercion, and maintaining objectivity. The common approach in research studies is to limit the relationship with participants to a professional relationship confined to the constructs of the research study. This approach allows both the researcher and the participant to maintain several degrees of separation. Objectivity therefore is easier to maintain, and coercion is readily avoided. However, the common model for research studies developed largely in urban-based areas with access to many resources. [21] In addition to resources, there may be public familiarity with the researchers, which may translate to trust. For many rural areas this approach would mean the only time participants are exposed to researchers are within the confines of the research study. Rural community members are often isolated from other communities due to geography and therefore community members become interdependent. [22] As a result, non-community members are viewed with suspicion and distrusted making research in rural communities more difficult for outside institutions. [22] In 1991, Murray and Keller suggested that the urban model of mental health service delivery was inappropriate to meet the needs of rural communities [21] and we are suggesting the urban model for research studies follows suit.

Rural communities are complex social systems that involve interdependent community members. The community structure and relationships in rural communities have deep cultural, familial, historical, and political roots that can influence the research study. [21] For instance, in Southern West Virginia, politeness is often held in high regard. Participants may be reluctant to tell a researcher "no" even if 
the participant is uncomfortable for fear of seeming impolite. Building a relationship and trust before beginning the research study could lead to an environment in which the participant is comfortable being honest potentially resulting in more accurate data and coercion avoidance. This approach goes against general practice particularly in psychological research, which suggests avoiding dual relationships as they can potentially lead to a loss in autonomy as participants may believe that certain behavior may disappoint the researcher or disrupt the relationship in some fashion. [22] While this may be the case in urban areas, maintaining distance in rural areas may not only be inappropriate, but may lead to a lack of care. [22] A dual relationship, although against the commonly accepted approach, becomes pertinent for the success of the research study. The patients are content expert and critical information is missed unless the patient perspective is relayed to the researcher. This is only possible if there is trust, which often stems from a dual relationship.

\subsection{Time Constraints and Budget Conflicts}

Mentioned previously, gaining a populations trust may consume a considerable amount of time and can result in budget conflicts. For example, the research project discussed was originally a 12-month project that included study start up procedures, conduction of the study itself, closing the study, and publishing the results. The original design included six total research visits for each of the 20 potential participants (Table 1). This plan was conducive with a small grant awarded for $\$ 25,000$ in which the only anticipated cost was travel, publication, and presentation costs. All other required materials were in the study team's possession prior to the design of this study. Once we began, it became clear that building a relationship and gaining the trust of the population was a mandatory step for our success. This process consumed almost 6 months of consistent engagement with the population before achieving recruitment progress, driving our timetable from a 12 -month project to a minimum of 18 months. During these six months, the researchers were present at the clinic once a week every week building a professional relationship with the participants. This relationship resulted in the participants providing pertinent information regarding the efficacy of the study. The provided information led to the protocol redesign that more than doubling our original budget. (Table 1) Most of the time constraints and budget conflicts discussed could have been avoided in the early planning stages of this study.

Table 1. Budget conflicts that resulted as a need for protocol redesign became apparent.

\begin{tabular}{lllll}
\hline & $\begin{array}{l}\text { Number of Study } \\
\text { Visits }\end{array}$ & $\begin{array}{l}\text { Number of } \\
\text { Participants }\end{array}$ & $\begin{array}{l}\text { Total Number of } \\
\text { Visits }\end{array}$ & $\begin{array}{l}\text { Total Study Visit } \\
\text { Round Trip Cost }\end{array}$ \\
\hline Original Design $_{\text {Including Pre-study visits }}{ }^{\text {a }}$ & 6 & 20 & 120 & $\$ 173.00$ \\
Redesign $^{\text {b }}$ & $6(+24)$ & 20 & 144 & $\$ 173.00$ \\
\hline
\end{tabular}

Table 2. Obstacles participants face in rural MAT programs that affect research study design.

\begin{tabular}{|c|c|c|c|}
\hline Obstacles & $\begin{array}{l}\text { Information provided } \\
\text { by Site Professionals }\end{array}$ & Information provided by Participants & Changes to Research Design \\
\hline Transportation & $\begin{array}{l}\text { Scarce, or unreliable } \\
\text { patient transportation }\end{array}$ & $\begin{array}{l}\text { Individuals providing transportation are unable or unwilling } \\
\text { to wait at the clinic } \\
\text { Multiple trips to clinic not feasible } \\
\text { Issues create limited time at clinic appointments }\end{array}$ & $\begin{array}{l}\text { Multiple, short study visits based around } \\
\text { existing clinic appointments was } \\
\text { utilized }\end{array}$ \\
\hline Time Constraints & $\begin{array}{l}\text { Multiple appointments } \\
\text { were not feasible }\end{array}$ & $\begin{array}{l}\text { Patients see physician, behavioral health, and the nurse } \\
\text { practitioner for urine drug screen in one appointment } \\
\text { Not enough time for all study visit tasks as well as other } \\
\text { scheduled tasks }\end{array}$ & $\begin{array}{l}\text { Limited to } 1-2 \text { study visit tasks per visit } \\
\text { Study tasks performed while participant } \\
\text { was waiting for regular appointment to } \\
\text { begin }\end{array}$ \\
\hline $\begin{array}{l}\text { Lack of } \\
\text { Understanding }\end{array}$ & $\begin{array}{l}\text { Participants were } \\
\text { unfamiliar with research } \\
\text { study logistics }\end{array}$ & $\begin{array}{l}\text { Participants needed more time to ask questions and receive } \\
\text { explanations of the study } \\
\text { Participants needed more information on what researchers do } \\
\text { compared to the clinicians they see regularly } \\
\text { Participants needed more time to make a decision and were } \\
\text { more apt to talk to their family first }\end{array}$ & $\begin{array}{l}\text { Integrated multiple discussions in group } \\
\text { and individual meetings before } \\
\text { approaching participants for consent } \\
\text { More time was allocated for the consent } \\
\text { process }\end{array}$ \\
\hline
\end{tabular}

Including pre-study visits: Study staff visited the clinic once a week for 6 months. Since study staff could engage the study population in one day a total of 24 visits rather than 24 visits per each of the 20 participants was required. As a result, 144 visits were required of study staff.
Redesign: The original 6 study visits were divided into multiple days resulting in 17 visits/patient to allow for completion of study tasks in addition to 24 pre-study visits. A total of 364 visits was required of study staff. 


\subsection{Constructing a Rural Research Schema}

The following is a more detailed account of how our trial design changed and the pressures that formulated our recommended approach. (Table 2)

\subsubsection{Transportation}

In the beginning, transportation was believed to be the largest obstacle for participants. To accommodate, the original design kept study visits to the minimum that would achieve study milestones and would occur on days when participants had to be at the test site for a previously scheduled clinic appointment. Participants had difficulty attaining transportation to and from the clinic and therefore we were attempting to minimize the number of required trips to the clinic; only 6 study visits would be required (figure 1). However, individuals providing transportation for the participants were oftentimes unable or unwilling to wait at the clinic for an extended period, nor were they able to return to the clinic later to pick up the participant. This issue created limited time for participants at the clinic. Due to limited time, 1-2 study tasks were completed per visit to accommodate participant schedule and needs breaking the original six study visits into 17 days (figure 2). This was only possible after the participant began to trust the researchers and felt comfortable enough to provide personal information to schedule study visits. After trust was established, participants would relay information freely and directly to the researchers rather than through a clinic staff member resulting in more accurate information and efficient scheduling. While straying from the distant observer method, a dual relationship maintained ethical conditions as study information remained exclusive between the participant and researcher.

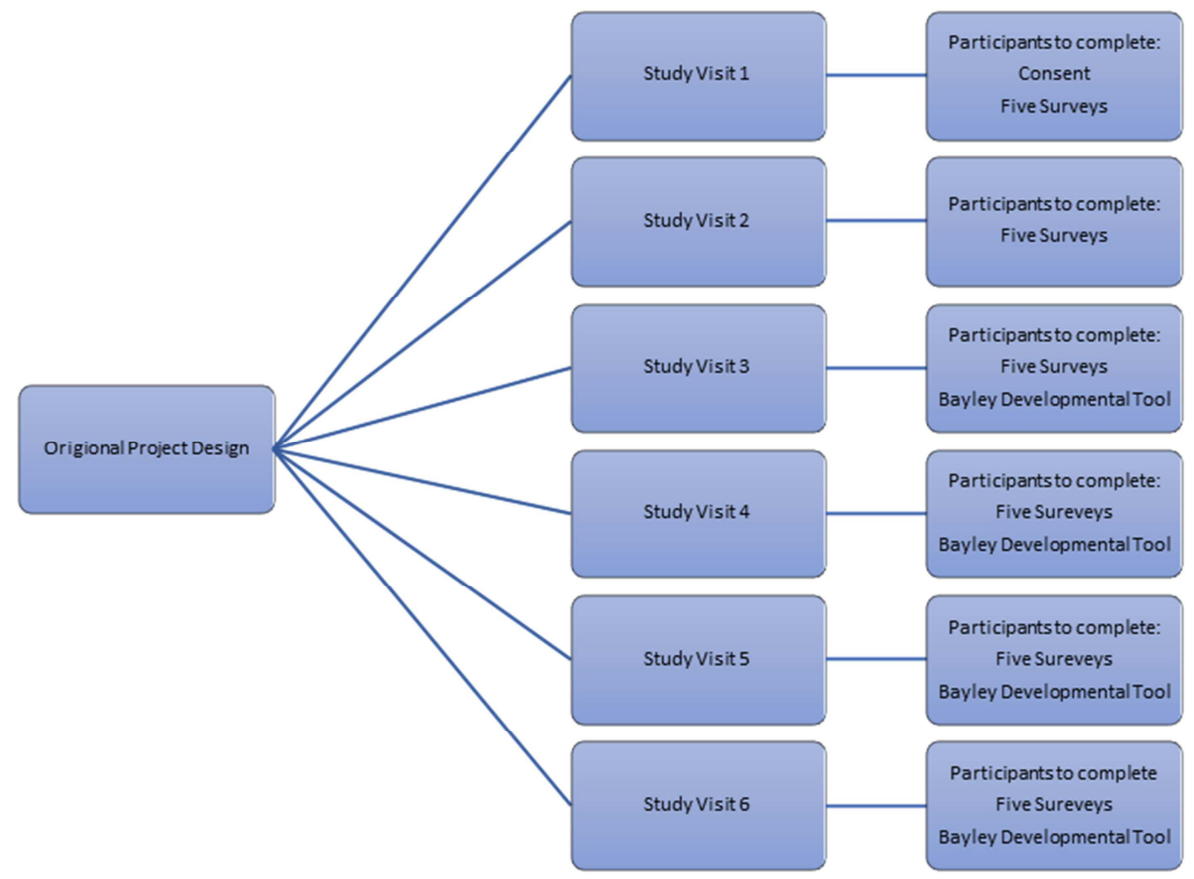

Figure 1. Schematic of the original project design.

\subsubsection{Time Constraints}

In the original design, participants would complete all the study visit needs in one setting at a set time with the researcher to eliminate the necessity for return visits. Study visit objectives included five maternal survey indices and the Bayley Developmental tool for their children. Although neither the survey indices or the developmental tool were lengthy or time consuming the participants simply did not have enough time to complete the study requirements, see their physician, see the psychologist for behavioral health and complete their urine drug screen in one setting. To correct this issue, we not only limited each visit to 1-2 tasks per visit but also eliminated a set time with the researcher. Study tasks were completed while the participant was waiting for an appointment to start or in between appointments. Eliminating a strict research visit schedule allowed us to achieve study milestones while avoiding additional stress and obstacles for the participants. This process was streamlined once a dual relationship began to form as participants felt comfortable approaching research staff to discuss any scheduling conflicts that would hinder study progress. Participants also began to approach researchers when the participants had free time in the clinic to complete study tasks.

\subsubsection{Lack of Understanding}

The population at the rural site had little experience with research studies and the research team allocated time to explain the project. The professionals at the health clinic began the conversation well before the project was initiated. However, once the research team initiated the study it was clear that participants had numerous questions regarding the study, the roll of researchers, the biology of substance abuse and medication assistant treatment. Elevating their concerns while allowing time to answer questions so the participants could fully understand was a lengthier process than anticipated. In addition, the informed consent process needed 
to be extended to allow participants time to make their decision and, in most cases, to discuss the decision with their families. To correct these issues, we allocated time to discuss the study and the researchers' rolls both in group sessions and individual sessions. These conversations continued for the entirety of the study. In addition to ongoing conversations, participants were given the opportunity to discuss their decision to participate with their families before consenting and intake into the study. Issues such as coercion and multiple layered relationships within projects can lead to difficult situations. Yet failure to take the time to construct relationships with trusted agencies and individual research participants can render data collection incomplete or impossible. As the conversations and questions about the research study flowed participants began to fully understand the study, the consenting process was more appropriate, and recruiting and retention efforts improved.

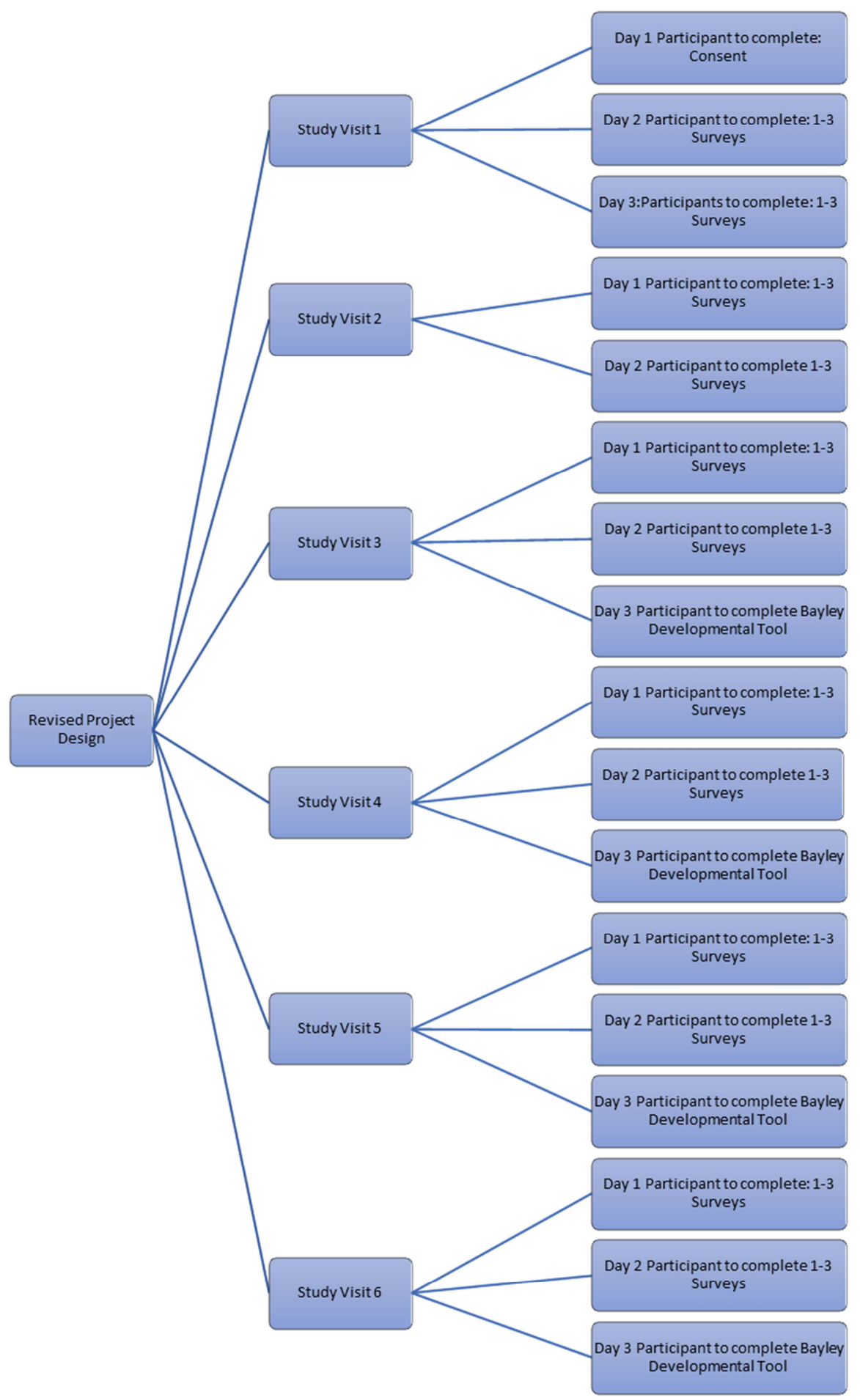

Figure 2. Schematic of the revised project design. 


\subsubsection{Health Needs}

Due to the nature of substance use this population has health needs that exceed treatment for OUD. A retrospective chart review to better under health need was conducted. In addition, an intake form addressing general health concerns was completed by participants. We did not anticipate the fear our research study would generate as participants began to consider the "additional" information collected. The pregnant women we hoped to recruit into the study did not understand the confidentiality requirements for researchers. These women were terrified of losing custody of their newborn babies and were not willing to agree to a study in which sensitive information about themselves and their substance abuse would be readily available to strangers. The nature of a close-knit community indicative of rural areas made it difficult for this population to trust researchers from outside of their community. An overall lack of understanding of post-natal events that would occur both in the hospital and after mom and baby were released from hospital care existed. This fear created an environment in which recruiting was all but impossible.

While the fear of losing custody created problems, it was not a unique situation in that women residing in more urban areas experience the same fears. However, fear of miscarriage was common among the pregnant women participating at the rural site. This population had witnessed or experienced miscarriage prior to their current pregnancy and were reluctant to change their normal routine for fear of spontaneous loss of the pregnancy. Even though this was an observational study with no interventions the fear of miscarriage was so overarching that recruitment became nearly impossible.

We immersed ourselves into group meetings and individual sessions when appropriate. We were present at the clinic weekly for extended periods of time which helped us build trust and report. Lengthy discussions were held about likely postnatal events, confidentiality requirements, and the steps taken to protect participant information. We were able to hold these discussions until the participants were comfortable with the information and revisited the conversations when necessary. This consistency was imperative to conducting the research study.

\section{Conclusion}

The complexities of studies with rural isolated populations including the need for greater funding could potentially result in these populations being underrepresented. This is exceptionally problematic when much of the area in the opioid epidemic represents rural, underserved, isolated areas. Some aspects thought to be driving forces of substance abuse, isolation for example, may be more prevalent in rural areas as compared to urban counterparts. Therefore, a more robust understanding of substance abuse may result from including rural areas in more research efforts. The logistical issues described could have been avoided had our research team immersed ourselves in the population before designing the study. Both the research team and the test site collaborators had experience with populations struggling with OUD however, it was clear that the rural population was vastly different from the urban population the researchers were accustomed despite similarities in genetics and culture. Collaborating with the staff provided an invaluable working relationship and important insight on how to approach research with the rural population. However, it was the impact of participant involvement, trust, open communication, and dual relationship that had the greatest effect on data collection and the success of the study. Once trust was established, the participants were enthusiastically provided information to better the logistics, became eager to participate and forthcoming with any problems and concerns they experienced. Not only did this allow us to redesign our protocol to be patient centric but it also allowed us to battle some common issues surrounding OUD such as the overall negative stigma helping not only the study population but the community as well.

\section{Recommendations}

Immersing oneself in a study population will allow the researcher to build trust and report with the population before attempting to recruit, creating a multilayered relationship that can rise some ethical concerns. While this multilayered relationship will allow the team to fully understand the population the research team must remain vigilant in maintaining participant autonomy to avoid coercion and other inappropriate situations. The recommended approach and the relationship focus we are suggesting is anti-theoretical to the typical research model. However, numbers in rural research studies are often too small to support statistics and this could be corrected by building relationships first rather than insisting upon several degrees of separation. This approach could potentially help recruitment and retention efforts while protecting populations from being alienated from research or skewing data.

\section{Resources}

1. Williams RL, Shelley BM, Sussman AL, clinicians RN. The marriage of community-based participatory research and practice-based research networks: can it work? -A Research Involving Outpatient Settings Network (RIOS Net) study. J Am Board Fam Med. 2009; 22 (4): 428-35.

2. Westfall JM, VanVorst RF, Main DS, Herbert C. Community-based participatory research in practice-based research networks. Ann Fam Med. 2006; 4 (1): 8-14.

3. Shippee ND, Domecq Garces JP, Prutsky Lopez GJ, Wang Z, Elraiyah TA, Nabhan M, et al. Patient and service user engagement in research: a systematic review and synthesized framework. Health Expect. 2015; 18 (5): 
1151-66.

4. Selby JV, Beal AC, Frank L. The Patient-Centered Outcomes Research Institute (PCORI) national priorities for research and initial research agenda. JAMA. 2012; 307 (15): 1583-4.

5. Mullins CD, Abdulhalim AM, Lavallee DC. Continuous patient engagement in comparative effectiveness research. JAMA. 2012; 307 (15): 1587-8.

6. Frank L, Forsythe L, Ellis L, Schrandt S, Sheridan S, Gerson J, et al. Conceptual and practical foundations of patient engagement in research at the patient-centered outcomes research institute. Qual Life Res. 2015; 24 (5): 1033-41.

7. Domecq JP, Prutsky G, Elraiyah T, Wang Z, Nabhan $M$, Shippee N, et al. Patient engagement in research: a systematic review. BMC Health Serv Res. 2014; 14: 89.

8. Boote JD, Twiddy M, Baird W, Birks Y, Clarke C, Beever D. Supporting public involvement in research design and grant development: a case study of a public involvement award scheme managed by a National Institute for Health Research (NIHR) Research Design Service (RDS). Health Expect. 2015; 18 (5): 1481-93.

\section{Abbreviations}

OUD, Opioid Use Disorder; NAS, Neonatal Abstinence Syndrome; MAT, Medication Assisted Treatment.

\section{Conflict of Interest}

The authors have no COI to disclose.

\section{Acknowledgements}

Funding for this work was provided by the Robert C. Byrd Center for Rural Health and the West Virginia Higher Education Policy Commission.

\section{References}

[1] Williams RL, Shelley BM, Sussman AL, clinicians RN. The marriage of community-based participatory research and practice-based research networks: can it work? -A Research Involving Outpatient Settings Network (RIOS Net) study. J Am Board Fam Med. 2009; 22 (4): 428-35.

[2] Westfall JM, VanVorst RF, Main DS, Herbert C. Community-based participatory research in practice-based research networks. Ann Fam Med. 2006; 4 (1): 8-14.

[3] Shippee ND, Domecq Garces JP, Prutsky Lopez GJ, Wang Z, Elraiyah TA, Nabhan $M$, et al. Patient and service user engagement in research: a systematic review and synthesized framework. Health Expect. 2015; 18 (5): 1151-66.

[4] Selby JV, Beal AC, Frank L. The Patient-Centered Outcomes Research Institute (PCORI) national priorities for research and initial research agenda. JAMA. 2012; 307 (15): 1583-4.
[5] Mullins CD, Abdulhalim AM, Lavallee DC. Continuous patient engagement in comparative effectiveness research. JAMA. 2012; 307 (15): 1587-8.

[6] Frank L, Forsythe L, Ellis L, Schrandt S, Sheridan S, Gerson J, et al. Conceptual and practical foundations of patient engagement in research at the patient-centered outcomes research institute. Qual Life Res. 2015; 24 (5): 1033-41.

[7] Domecq JP, Prutsky G, Elraiyah T, Wang Z, Nabhan M, Shippee N, et al. Patient engagement in research: a systematic review. BMC Health Serv Res. 2014; 14: 89.

[8] Boote JD, Twiddy M, Baird W, Birks Y, Clarke C, Beever D. Supporting public involvement in research design and grant development: a case study of a public involvement award scheme managed by a National Institute for Health Research (NIHR) Research Design Service (RDS). Health Expect. 2015; 18 (5): 1481-93.

[9] Sharma NS. Patient centric approach for clinical trials: Current trend and new opportunities. Perspect Clin Res. 2015; 6 (3): 134-8.

[10] Lim SS, Kivitz AJ, McKinnell D, Pierson ME, O'Brien FS, Simulating clinical trial visits yields patient insights into study design and recruitment. Patient Prefer Adherence. 2017; 11: 1295-307.

[11] Murthy RS. National Mental Health Survey of India 20152016. Indian Journal of Psychiatry. 2017; 59 (1): 21-6.

[12] Villapiano NL, Winkelman TN, Kozhimannil KB, Davis MM, Patrick SW. Rural and Urban Differences in Neonatal Abstinence Syndrome and Maternal Opioid Use, 2004 to 2013. JAMA Pediatr. 2017; 171 (2): 194-6.

[13] Sigmon SC. Access to treatment for opioid dependence in rural america: Challenges and future directions. JAMA Psychiatry. 2014; 71 (4): 359-60.

[14] Oser CB, Harp KL, O'Connell DJ, Martin SS, Leukefeld CG. Correlates of participation in peer recovery support groups as well as voluntary and mandated substance abuse treatment among rural and urban probationers. J Subst Abuse Treat. 2012; 42 (1): $95-101$.

[15] Brown JD, Goodin AJ, Talbert JC. Rural and Appalachian Disparities in Neonatal Abstinence Syndrome Incidence and Access to Opioid Abuse Treatment. J Rural Health. 2017.

[16] Eastwood J, Ogbo FA, Hendry A, Noble J, Page A, Early Years Research G. The Impact of Antenatal Depression on Perinatal Outcomes in Australian Women. PLoS One. 2017; 12 (1): e0169907.

[17] Hoffman C, Dunn DM, Njoroge WFM. Impact of Postpartum Mental Illness Upon Infant Development. Current Psychiatry Reports. 2017; 19 (12): 100.

[18] Logan BA, Brown MS, Hayes MJ. Neonatal abstinence syndrome: treatment and pediatric outcomes. Clin Obstet Gynecol. 2013; 56 (1): 186-92.

[19] Kocherlakota P. Neonatal abstinence syndrome. Pediatrics. 2014; 134 (2): e547-61.

[20] Kinsella MT, Monk C. Impact of maternal stress, depression and anxiety on fetal neurobehavioral development. Clin Obstet Gynecol. 2009; 52 (3): 425-40. 
[21] Helbok CM, Marinelli RP, Walls RT. National survey of ethical practices across rural and urban communities. Professional Psychology: Research and Practice. 2006; 37 (1): 36-44.
[22] Campbell CD, Gordon MC. Acknowledging the inevitable: Understanding multiple relationships in rural practice. Professional Psychology: Research and Practice. 2003; 34 (4): 430. 